\title{
Mapping Evidence of the Power Relations Between Domestic Workers and Their Employers in Sub- Saharan Africa: a Scoping Review Protocol
}

Thobeka Ntini ( $\nabla$ ntinithobeka@gmail.com )

University of Zululand Faculty of Arts https://orcid.org/0000-0002-3457-5211

Delarise Mulqueeny

University of Zululand Faculty of Arts

Vishanthie Sewpaul

University of KwaZulu-Natal School of Applied Human Sciences

\section{Protocol}

Keywords: power relations, domestic workers, employers, sub-Saharan Africa

Posted Date: December 20th, 2021

DOl: https://doi.org/10.21203/rs.3.rs-1130557/v1

License: (c) (i) This work is licensed under a Creative Commons Attribution 4.0 International License.

Read Full License 


\section{Abstract \\ Background}

Across various intersectional lines, including race, class and gender, domestic work is profoundly exploitative than other comparable occupations. The private household, within which domestic workers (DWs) work and function, provides for a space of complex and nuanced dynamics of power. According to the International Labour Organization there are more than 65 million DWs in the world, and Africa is the third largest employer of DWs, with more than 5.2 million DWs reported. The inception of the Domestic Workers Convention (No. 189) (C189) in 2011 sought to protect DWs from all forms of exploitation and discrimination in the workplace, the convention brought global attention to the violation of their human rights and inequalities within the domestic work sector. Although there are more than 5.2 million DWs in Africa, 39/46 countries in sub-Saharan Africa (SSA) have not ratified this convention. Due to the extremely low ratification of the $\mathrm{C} 189$ and scanty evidence on the power relations between DWs and their employers in SSA, this scoping review is relevant to detect the extent and characteristics of domestic work in SSA since the introduction of C189 in September 2011.

\section{Methods}

The literature that will be included in this scoping review are published peer-reviewed articles, grey literature from relevant departmental websites, humanitarian organisations and theses. Electronic searches of databases and search engines such as Google, Google Scholar, EBSCOhost, EBSCO Discovery Service, Scopus, World Bank and International Labour Organization (ILO) for literature published between September 2011-2021. Other search engines will include screening citations and references of appearing literature within the stipulated time period. All retrieved literature will be exported to an Endnote X9 library. Duplicate documents will be deleted prior to commencement of title screening. An adapted Mixed Method Appraisal Tool (MMAT), developed in a Google form, will be used by two reviewers to quality assess and describe all included studies (qualitative, mixed methods and quantitative).

\section{Discussion}

We anticipate mapping relevant literature on the power relations between domestic workers and their employers in sub-Saharan Africa. Once analysis and summary is finalised, the data will be useful to guide future research.

\section{Background}

Domestic work is one of the oldest occupations that is considered as informal and vulnerable globally and it is characterised by the intersectionality of race, class, gender, migration, ethnicity and citizenship 
(1). Domestic workers (DWs) are individuals, male or female, who perform domestic work or duties in or for households that include cleaning, cooking and caring in or for household/s, and they may reside at the employer's household or commute regularly (2). Private households that are the primary workplaces for DWs constitute spaces of complex power relations and dynamics where inequalities and oppression are often produced and reproduced (3). The International Labour Organisation (ILO) (2018) estimates that 70.1 million DWs are employed across 176 countries globally with Africa being the third largest employer of DWs. The African continent accounts for an estimated 10 million DWs with the industry being primarily dominated by black African women from disadvantaged backgrounds (4). An ILO report states that informal and vulnerable employment constitutes $74 \%$ of women's employment in sub-Saharan Africa (SSA), while men make up $61 \%$ in this industry (5).

For decades, the recognition of and protection of DWs labour rights and protection were non-existent internationally and in SSA. However, the ILO has made significant strides for the socio-economic justice of DWs by setting international working standards. Despite this, domestic work remains a site for different forms of oppression as the work can be dehumanising and labour intensive. DWS are grossly underpaid for their long hours of work, and often without concise terms of employment and outside the scope of labour rights. These conditions position them as subjects of irregular working conditions and exploitation which are integral concerns for social justice and human rights (6). Hence, the introduction of the Domestic Workers Convention (No. 189) (C189) in 2011, which sought to protect DWs from all forms of exploitation and discrimination in the workplace, brought global attention to the violation of their human rights and inequalities within this sector. However, several SSA countries have not ratified this convention (7) and evidence for detecting the extent and characteristics of domestic work in Africa is inadequate and insufficient.

Domestic workers are included in the United Nations Sustainable Development Goals (SDG's), particularly SDG 5: Gender Equality, SDG 8: Decent work and economic growth and SDG 10: Reduced inequality (8). These SDGs are fundamental in informing the development of legislations, policies, guidelines and advocacy frameworks aimed at promoting human rights and equality of all citizens, including DWs. Engaging in a more nuanced analysis of power relations between DWs and their employers is a critical step toward reconstructing and redefining the positionality of DWs. Thus, a scoping review aimed at mapping and synthesising existing evidence on the power relations between DWs and their employers in SSA is relevant. It could assist in improving the knowledge base of domestic work and influence policy development and pragmatic human rights solutions on labour matters that could ameliorate tensions and inequalities in DW and employer relationships. The review will focus on literature published from September 2011 to 2021. The selected time period is motivated by the existence of C189 that was introduced in 2011. This time frame is particularly important for scoping and synthesising evidence on the power relations between DWs and their employers in SSA as there is only seven (Namibia, South Africa, Madagascar, Mauritius, Sierra Leone and Guinea) out of 46 SSA countries that have ratified this convention.

\section{Objective}


The review will be guided by the following objective:

a. To systematically map existing evidence on the power relations between DWs and their employers in SSA.

The results of the study could benefit policy makers, domestic workers, employers of domestic workers and relevant regulatory and umbrella bodies to dismantle any inequalities and improve domestic workers work and personal lives.

\section{Methods}

Domestic work in SSA is a broad topic as DWs' experiences may differ across the various countries in SSA depending on the local laws and ratifications of international conventions and treaties. Hence, a scoping review was chosen to map evidence to answer our research question by systematically and rigorously searching, selecting, and integrating existing literature on the power relations between DWs and their employers in SSA (9). This scoping review will serve as a solid basis for a comprehensive interrogation of available literature in SSA, and will serve as a precursor to a systematic review on the phenomenon of domestic work.

The screened literature will include published qualitative, quantitative, and mixed-methods studies that will identify knowledge gaps on the power relations between DWs and their employers. We will use the five stages of Arksey and O'Malley's six stages, in conjunction with developments made by Levac and colleagues. The five stages comprise of (i) identifying the research question, (ii) identifying relevant literature, (iii) study selection, (iv) data charting, and (v) collating, summarising and reporting the results $(10,11)$.

This protocol will adhere to rigorous standards and address all the critical steps required by following the PRISMA ScR (Preferred Reporting Items for Systematic Review and meta-Analysis Extension for Scoping Reviews) checklist (12-14).

The scoping review protocol has been registered on the Open Science Framework (OSF) on October 13, 2021 in the preregistration category and can be accessed using the link: https://osf.io/ujmnx .

\section{(i) Identifying the research question}

To ensure that the study selection addresses the research question, the Population, Concept, Context (PCC) criteria format from the Joanna Briggs Institutes will be used (see Table 1) (15).

\section{Primary question}

What evidence exists on the power relations between domestic workers and their employers in SSA?

\section{Secondary question}


- What factors contribute to inequalities in the relations between DWs and their employers in SSA?

\section{Eligibility criteria}

The eligibility of relevant studies that address the research question were guided by the population, Concept, Context (PCC) framework as presented in the following table:

Table 1

PCC framework

\section{Population Domestic workers \\ Refers to individuals older than 18 years, who perform domestic work or duties in or for households that include cleaning, cooking and caring in or for household/s, and reside at the employer's household or commute regularly (2).}

Concepts Power relations between domestic workers and their employers

Refers to unequal or unfair relationships between domestic workers (DWs) and their employers, where either DWs or/and employers feel powerless or privileged (16).

Context Sub-Saharan Africa: Includes 46 countries that are geographically located in the south of the Saharan desert in Africa and exclude eight countries, namely, Djibouti, Algeria, Egypt, Libya, Morocco, Somalia, Sudan and Tunisia (17).

\section{(ii) Identifying relevant studies}

This scoping review will chart available, relevant literature to map the power relations between DWs and their employers in SSA, peer-reviewed articles and reliable grey literature (government gazettes, reports, global and national statistics, conventions, conference papers, theses and dissertations) between September 2011 and 2021. Electronic searches of databases will include EBSCO Discovery Service, Scopus and EBSCOhost (namely, Academic Search Ultimate, Africa-Wide Information, APA PsycBooks, APA Psyclnfo, APA PsycTests, Audiobook Collection (EBSCOhost), eBook Collection (EBSCOhost), eBook Open Access (OA) Collection (EBSCOhost), ERIC, GreenFILE, Newspaper Source, Newswires, Open Dissertations, PEP Archive and Sociology Source Ultimate). Other search engines that will be used are: Google, Google Scholar including screening citations and references of appearing literature, the ILO, World Health Organisation (WHO) and World Bank within the stipulated time period. Literature identified will be exported to an Endnote X9 library and duplicated literature will be removed before title screening commences.

All included literature on the power relations between DWs and their employers in SSA will be guided by the inclusion criteria (see study selection below), our research questions and the study population of DWs 18 years and older who live and work in SSA. 
The reliability and viability of this scoping review was tested through a pilot search conducted on one database by two screeners (TN, DM) using the keywords: "Domestic workers", "power relations", "power dynamics", "control", "sub-Saharan Africa" and "Africa". The pilot search also made the use of Boolean terms 'AND', 'NOT' and 'OR' to separate search terms. The results thereof are presented in Table 2. The strategy to search for relevant literature will be guided by an experienced librarian. Studies without a publication date will not be included to eliminate any discrepancies in the findings, this is particularly important because the specified time period starts from when the $\mathrm{C} 189$ was introduced. Where the journal articles or books are inaccessible, the librarian will be contacted to explore alternatives to access literature such as interlibrary loans.

Table 2

Pilot database search results

\begin{tabular}{|c|c|c|c|}
\hline Keywords & Search Date & Search Engine & $\begin{array}{l}\text { No. } \\
\text { retrieved }\end{array}$ \\
\hline $\begin{array}{l}\text { "power relations" or power or "power dynamics" or } \\
\text { control } \\
\text { AND }\end{array}$ & \multirow[t]{9}{*}{$2021 / 10 / 29$} & \multirow[t]{9}{*}{$\begin{array}{l}\text { Academic Search } \\
\text { Complete on } \\
\text { EBSCOHost }\end{array}$} & \multirow[t]{9}{*}{466} \\
\hline & & & \\
\hline $\begin{array}{l}\text { "domestic workers" or "household employees" or } \\
\text { caregivers or nannies or maids }\end{array}$ & & & \\
\hline AND & & & \\
\hline employer OR "employer employee relationship" & & & \\
\hline AND & & & \\
\hline Africa OR sub saharan Africa OR African countries & & & \\
\hline NOT & & & \\
\hline $\begin{array}{l}\text { Middle East OR Djibouti OR Algeria OR Egypt OR } \\
\text { Libya OR Morocco OR Somalia OR Sudan OR } \\
\text { Tunisia }\end{array}$ & & & \\
\hline
\end{tabular}

\section{(iii) Study selection}

To ensure that relevant literature sufficiently addresses the research question in this scoping review, the inclusion and exclusion criterion were formulated and appear below.

\section{Inclusion Criteria}

The following criteria will ensure the included literature pertains to the scoping review's research objectives: 
- Studies relating to the power relations between DWs and their employers in the 46 countries in SSA.

- Literature that includes employers who live and employ DWs in SSA.

- Literature that includes DWs who work in SSA.

- Grey literature (Government reports, policy statements, conference proceedings, theses and dissertations) from September 2011 to 2021.

- Literatures on the topic published between September 2011 to 2021. The motivation for the time period is due to the inception of the $\mathrm{C} 189$.

\section{Exclusion Criteria}

This scoping review will exclude:

- Literature that includes DWs from SSA who work outside of SSA.

- Literature that includes DWs from outside SSA who work outside of SSA.

- Literature that includes employers who live in SSA but employ DWs outside of SSA.

- Literature that includes child (under 18 years) DWs, who perform paid domestic work duties.

- Literature published prior to September 2011.

- Studies conducted outside of the 46 SSA countries.

\section{(iv) Charting of data}

To create a descriptive summary from the retrieved literature, a Google form will be used to develop an electronic data charting form. It will include all relevant information and populated with data extracted from each selected piece of literature that relates to the research objective. To ensure that the form remains relevant, accurate and timeous, the data charting form (Table 3 ) will be constantly reviewed, to ensure it answers the research question. The data charting form includes the discipline within which that literature has been published to observe and report on the extent that certain disciplines cover the phenomenon of power relations in domestic work. 
Table 3

Data charting table

\begin{tabular}{|l|}
\hline Author and Year of publication \\
\hline Discipline of the journal \\
\hline Title \\
\hline Study Aim/s \\
\hline Study design \\
\hline Study setting \\
\hline Study population \\
\hline Country of population \\
\hline Experiences of unequal or unfair relationship \\
\hline Relevant findings \\
\hline Key conclusions of article \\
\hline
\end{tabular}

\section{(v) Collating, summarizing and reporting the results}

Findings from retrieved literature will be analysed thematically as stipulated by Braun and Clarke's thematic framework (18). Atlas.ti 8 data analysis software will be used to identify, analyse and report themes within data (19). Literature will then be arranged according to themes that developed within the charted data during coding (20). All participating researchers (primary author and screeners) in the study will jointly formulate the themes. The objective of the study, which is to map evidence of the power relations between DWs and their employers in SSA, will guide the examination of the emerging themes. This will be in relation to the rigour and validity of the research question. Where discrepancies are apparent, a third screener will resolve discrepancies. As previously done by Mulqueeny and colleagues, the screeners and authors will have regular reflexive meetings to reach consensus which will in turn minimise biases and inconsistencies (21).

\section{Quality appraisal}

For quality assessments and descriptions of all included studies, an adapted 2018 version of Mixed Method Appraisal Tool (MMAT) will be used (22). To provide a rigorous account of the validity and reliability of reported findings, all sources of included and excluded data will follow a methodical and logical approach that ensures the evidence synchronises with the study objective, suitable methodologies and sampling. Additionally, proper and unbiased data interpretation, findings, discussion, conclusions and recommendations of all literature will be included. Littlewood, Chance-Larsen and McLean (2010) suggest that "inadequate attention to key design features including sample size, allocation and level of 
blinding" may increase inaccurate conclusions (23). Therefore, the quality of collected literature will be appraised through ratings from high average $(100 \%)$ to above average $(75 \%)$, average $(50 \%)$ and lowquality (25\%) research evidence (24).

\section{Discussion}

Subsequent to numerous socio-economic-political changes occurring in SSA, and international conventions and legislation promulgated to protect domestic workers' rights, domestic work still remains a site for various forms of oppression (25). Historically, DWs have been described as "trapped within a tightly woven structure of constraints" (26). In contemporary times, it continues to be the typical analysis of the power trip of domestic worker employers, and it undeniably bears reality as "the space domestic workers occupy is still largely marginal" (27). However, literature and mass media often discuss the dynamism of power as one-directional, while ignoring the complexities and entanglement of the relationship. Without denying that domestic work is profoundly exploitative than other comparable occupations (28), the proposed scoping review will produce findings that comprehensively identify and describe the power relations between DWs and their employers. This is to identify knowledge gaps and highlight multiple truths leading to a more nuanced analysis of power in DW-employer relations across SSA.

Findings generated from this scoping review will be valuable to labour and humanitarian organisations, social justice and human rights organisations, community-based organisations (CBOs), nongovernmental organisations (NGOs), policies, labour conventions and law, and inform awareness campaigns and civic engagement. Moreover, it could encourage diverse scholarship, since power relations between DWs and their employers implicate many inequalities and exploitation that various disciplines aim to eradicate. Additionally, domestic work is not an isolated phenomenon but of interest to various disciplines. It could drive the needed engagement and solution-focused dialogues, which can reduce inequalities as clearly pronounced in SDG 5: Gender Equality and SDG 10: Reduced inequality (8). The reviews findings could offer recommendations for future research and practices, be published in peer-reviewed journals, assist governmental reports and policy briefs, and be presented at international and national conferences as well as seminars. The findings can contribute to the existing body of knowledge for teaching and learning purposes in disciplines where informal economies and vulnerable occupations are prioritised.

\section{Limitations}

A scoping review with time restrictions could exclude valuable data on the power relations between DWs and their employers, for countries that have reported research evidence prior to the inception of C189.

\section{Abbreviations}


DWs Domestic workers

ILO International Labour Organization

SDGs Sustainable Development Goals

SSA Sub-Saharan Africa

WHO World Health Organization

\section{Declarations}

\section{Acknowledgement}

The authors express their gratitude to United Nations Children's Fund (UNICEF), Future Africa project and University of Pretoria for support of this study. The authors also express gratitude to the National Research Foundation that sponsors the main author for her doctoral study. Ms. Kabelo Kgarosi, Faculty of Health librarian in the University of Pretoria and Ms. Faith Nsele, Faculty of Arts librarian in the University of Zululand are acknowledged for their assistance with searches and databases.

\section{Author's Information}

Ms Thobeka Ntini

Affiliation: ${ }^{1} \mathrm{~A}$ PhD candidate at the University of KwaZulu-Natal in the Discipline of Social Work.

${ }^{2}$ A lecturer in the Department of Social Work, Faculty of Arts, University of Zululand, Private Bag X1001, KwaDlangezwa, 3886, South Africa.

Email: ntinithobeka@gmail.com / NtiniT@unizulu.ac.za

Dr. Delarise Maud Mulqueeny,

Affiliation: ${ }^{1}$ Senior Lecturer in the Department of Social Work, Facultyof Arts, University of Zululand, Private Bag X1001, KwaDlangezwa, 3886, South Africa.

${ }^{2}$ Discipline of Public Health Medicine, School of Nursing and Public Health, University of KwaZulu-Natal, South Africa.

Email: delarisemulqueeny@gmail.com / mulqueenyd@unizulu.ac.za

Prof. Vishanthie Sewpaul 
Affiliation: Emiterus Professor in the Discipline of Social Work, School of Applied Human Sciences, 32 Mazisi Kunene Ave, Howard College Campus, Gate 1, Durban 4001, South Africa.

Email: sewpaulv@ukzn.ac.za

\section{Corresponding Author's details}

Ms. T. Ntini,

\section{Affiliation:}

${ }^{1} \mathrm{~A}$ PhD candidate at the University of KwaZulu-Natal, South Africa.

${ }^{2}$ Lecturer in the Department of Social Work, Faculty of Arts, University of Zululand, Private Bag X1001, KwaDlangezwa, 3886, South Africa.

Physical Address: Room 5, Faculty of Arts Building, 1 Main Road, Vulindlela KwaDlangezwa, Zululand, 3886, South Africa.

Email: ntinit@unizulu.ac.za /ntinithobeka@gmail.com

\section{Funding}

Not applicable.

\section{Availability of data and materials}

The published scoping review protocol will include all the data generated and analyzed in this study is available upon request.

\section{Authors contributions}

The protocol was conceptualised by TN. All three authors were jointly involved in the development, writing and approval of the protocol. Three researchers will be involved in the actual study, abstract and full article screening process with the assistance of a librarian.

\section{Competing interests}

The authors have no competing interests.

\section{Consent for application}

Not applicable

\section{Ethical approval and participants consent}




\section{References}

1. May VM. Pursuing Intersectionality, Unsettling Dominant Imaginaries. New York: Routledge; 2015.

2. The Domestic Workers Convention, (C 189) (2011).

3. Paraciani R, Rizza, R. When the workplace is the home: Labour inspectors' discretionary power in the field of domestic work - an institutional analysis. Journal of Public Policy. 2021;1 (41):1-16.

4. Organization IL. Care work and care jobs for the future of decent work. International Labour Organization; 2018.

5. Organization IL. Five facts about informal economy in Africa. 2021 [Available from: https://www.ilo.org/africa/whats-new/WCMS_377286/lang-en/index.html.

6. Fisher $\mathrm{O}$. The Impact of Micro and Macro Level Factors on the Working and Living Conditions of Migrant Care Workers in Italy and Israel-A Scoping Review. 2021;18(2):420.

7. Organization IL. Ratifications of C189 - Domestic Workers Convention, 2011 (No. 189) 2021 [Available from: https://www.ilo.org/dyn/normlex/en/f? p=NORMLEXPUB:11300:0::NO::P11300_INSTRUMENT_ID:2551460.

8. UN. The sustainable development agenda 2019 [Available from: https://www.un.org/sustainabledevelopment/development-agenda/.

9. Munn Z, Peters MD, Stern C, Tufanaru C, McArthur A, Aromataris EJBmrm. Systematic review or scoping review? Guidance for authors when choosing between a systematic or scoping review approach. 2018;18(1):1-7.

10. Arksey H, O'Malley LJljosrm. Scoping studies: towards a methodological framework. 2005;8(1):1932.

11. Levac D, Colquhoun H, O'Brien KKJIs. Scoping studies: advancing the methodology. 2010;5(1):1-9.

12. Page MJ, McKenzie JE, Bossuyt PM, Boutron I, Hoffmann TC, Mulrow CD, et al. The PRISMA 2020 statement: an updated guideline for reporting systematic reviews. 2021;372.

13. Boers MJH. Graphics and statistics for cardiology: designing effective tables for presentation and publication. 2018;104(3):192-200.

14. Mayo-Wilson E, Li T, Fusco N, Dickersin K, methods MiJRs. Practical guidance for using multiple data sources in systematic reviews and meta-analyses (with examples from the MUDS study). 2018;9(1):2-12.

15. on JBIJAA. The Joanna Briggs Institute reviewers' manual 2015: Methodology for JBI scoping reviews. 2015. 2019;16.

16. Veremenko VAJRJoRH. "Powerless power": The status of female domestic workers in Russia in the second half of the 19th-early 20th century. 2019;18(2):320-54. 
17. Kampala L. What is sub-Saharan Africa? 2021 [Available from: https://www.economist.com/theeconomist-explains/2019/03/07/what-is-sub-saharan-africa.

18. Clarke V, Braun V. Thematic analysis. Encyclopedia of critical psychology: Springer; 2014. p. 1947-52.

19. Friese S. Qualitative data analysis with ATLAS. ti: Sage; 2019.

20. Bryman A. Social research methods: Oxford university press; 2016.

21. Mulqueeny DM, Nkabini SM, Mashamba-Thompson TPJSr. School-going transgender youths' experiences at health care facilities: a systematic scoping review protocol. 2020;9(1):1-7.

22. Hong QN, Fàbregues S, Bartlett G, Boardman F, Cargo M, Dagenais P, et al. The Mixed Methods Appraisal Tool (MMAT) version 2018 for information professionals and researchers. 2018;34(4):28591.

23. Littlewood C, Chance-Larsen K, McLean SMJIJoP, Rehabilitation. Quality appraisal as a part of the systematic review: a review of current methods. 2010;1(1):53-8.

24. Mulqueeny DM, Nkabini SM, Pokiya MHJSr. Mapping evidence of depression in HIV-seropositive MSM in sub-Saharan Africa: a scoping review protocol. 2021;10(1):1-8.

25. Donald FM, Mahlatji LJJopiA. Domestic workers' experiences of power and oppression in South Africa. 2006;16(2):205-13.

26. Cock J. Maids \& madams: A study in the politics of exploitation: Ravan Press; 1980.

27. Mahali AJJoAMS. Maid to serve:'Self-fashioning'and the domestic worker trope in contemporary South Africa. 2016;8(2):127-43.

28. Collins PH. Black feminist thought: Knowledge, consciousness, and the politics of empowerment: routledge; 2002.

\section{Figures}

\section{Figure 1}

PRISMA ScR Flowchart shows the literature search and study selection processes (12). 